\title{
Promoting Axon Regeneration in the Adult CNS by Modulation of the PTEN/mTOR Pathway
}

\author{
Kevin Kyungsuk Park ${ }^{*}$, Kai Liu ${ }^{*}$, Yang Hu ${ }^{*}$, Patrice D. Smith ${ }^{*}$, Chen Wang, Bin Cai, Bengang \\ $\mathrm{Xu}$, Lauren Connolly, loannis Kramvis, Mustafa Sahin, and Zhigang $\mathrm{He}^{\dagger}$ \\ F. M. Kirby Neurobiology Center, Children's Hospital, and Department of Neurology, Harvard \\ Medical School, 300 Longwood Avenue, Boston, MA 02115, USA.
}

\begin{abstract}
The failure of axons to regenerate is a major obstacle for functional recovery after central nervous system (CNS) injury. Removing extracellular inhibitory molecules results in limited axon regeneration in vivo. To test for the role of intrinsic impediments to axon regrowth, we analyzed cell growth control genes using a virus-assisted in vivo conditional knockout approach. Deletion of PTEN (phosphatase and tensin homolog), a negative regulator of the mammalian target of rapamycin (mTOR) pathway, in adult retinal ganglion cells (RGCs) promotes robust axon regeneration after optic nerve injury. In wild-type adult mice, the mTOR activity was suppressed and new protein synthesis was impaired in axotomized RGCs, which may contribute to the regeneration failure. Reactivating this pathway by conditional knockout of tuberous sclerosis complex 1, another negative regulator of the mTOR pathway, also leads to axon regeneration. Thus, our results suggest the manipulation of intrinsic growth control pathways as a therapeutic approach to promote axon regeneration after CNS injury.
\end{abstract}

\begin{abstract}
Axons do not regenerate after injury in the adult mammalian central nervous system (CNS), a phenomenon attributed to two properties of the adult CNS, the inhibitory extrinsic environment and a diminished intrinsic regenerative capacity of mature CNS neurons (1-4). Neutralization of the extracellular molecules identified as axon regrowth inhibitors allows only a limited degree of axon regeneration in vivo (5-7). Therefore, intrinsic mechanisms are likely to be important in controlling the process of axon regeneration. A hint about possible mechanisms of neuronal regenerative ability comes from the evolutionarily conserved molecular pathways that control cellular growth and size. For most cell types, specific mechanisms are necessary to prevent cellular overgrowth upon the completion of development (8). Because many of these molecules are often expressed in postmitotic mature neurons, we hypothesized that they may contribute to the diminished regenerative ability in adult CNS neurons.
\end{abstract}

To circumvent the problem that germline knockout of individual cell growth control genes often results in compromised viability in mice, we designed a strategy based on intravitreal injection of adeno-associated viruses expressing Cre (AAV-Cre) in adult mice. This procedure resulted in the expression of Cre in more than $90 \%$ of retinal ganglion cells (RGCs) and few other non-RGC cells, as indicated in two reporter lines (fig. S1, A and B). We thus injected AAV-Cre into the vitreous body of different adult floxed mice, including $\mathrm{Rb}^{\mathrm{f} / \mathrm{f}}(9), \mathrm{P} 53^{\mathrm{f} / \mathrm{f}}(9)$, $\mathrm{Smad}_{4}^{\mathrm{f} / \mathrm{f}}(10), \operatorname{Dicer}^{\mathrm{f} / \mathrm{f}}(11), \mathrm{LKB} 1^{\mathrm{f} / \mathrm{f}}(12)$, and PTEN ${ }^{\mathrm{f} / \mathrm{f}}(13)$. At 2 weeks after AAV injection

\footnotetext{
*To whom correspondence should be addressed. E-mail: zhigang.he@ childrens.harvard.edu.

These authors contributed equally to this work.

Supporting Online Material

www.sciencemag.org/cgi/content/full/322/5903/963/DC1
} 
(fig. S1C), these animals were subjected to an optic nerve crush procedure (14). Axon regeneration was assayed by examining axonal fibers labeled with the anterograde tracer, cholera toxin $\beta$ (CTB), in the optic nerve sections across the lesion site. Neuronal survival was also estimated by whole-mount staining of the retina with immunostaining with an antibody to $\beta$-III tubulin (TUJ1) or by prelabeling with FluoroGold injected to the superior colliculi.

Among the different mouse lines examined, those with a PTEN (phosphatase and tensin homolog) deletion showed the largest effects on both neuronal survival and axon regeneration. In all PTEN ${ }^{\mathrm{f} / \mathrm{f}}$ conditional mutants injected with AAV-Cre, but not with control AAV-GFP (green fluorescent protein), RGCs displayed a significant increase in survival as indicated by TUJ1 staining (Fig. 1F and fig. S1D). Similar extents of neuronal survival were observed by prelabeling RGCs with FluoroGold (fig. S2). In addition, robust long-distance axon regeneration (Fig. 1, A to E, and fig. S3, A and B) was observed at 14 days after injury. We repeated the AAV-Cre experiments in another set of PTEN ${ }^{\mathrm{f} / \mathrm{f}}$ mice, as well as AAV-GFP injection as controls, and observed similar results. Quantification showed that $\sim 45 \%$ of PTENdeleted RGCs survived 2 weeks after injury, in comparison to $20 \%$ in controls. Of the surviving RGCs, $\sim 8$ to $10 \%$ showed regeneration of their lesioned axons beyond $0.5 \mathrm{~mm}$ distal to the lesion epicenter; some extended beyond $3 \mathrm{~mm}$ (Fig. 1, A and E, and fig. S3, A and B). These fibers continued to regenerate along the optic nerve over time. At 4 weeks after injury, some regenerating fibers extended to the area of the optic chiasm (Fig. 1, B, D, and E, and fig. S3, C and D). Among the other mouse lines tested, only p53-deleted RGCs showed increased neuronal survival but no axon regeneration (fig. S1, D and E), consistent with the notion that inducing neuronal survival is not sufficient to allow axon regeneration (15). Thus, PTEN deletion likely acts upon intrinsic mechanisms other than survival to promote axon regeneration after injury.

We next examined the time course of axon elongation of PTEN-deleted RGCs after injury. At the lesion site, a glial response occurred 1 to 3 days after crushing, as indicated by the upregulation of chondroitin sulfate proteoglycan (CSPG) expression (fig. S4, A and B) and macrophage infiltration (fig. S5). However, glial fibrillary acidic protein (GFAP)-positive astrocytes are largely excluded from the lesion sites, and CSPG signals returned to low levels by 7 days after injury (fig. S4C). At 1 day post-crush (dpc), injured optic nerve fibers terminate at the proximal end of the crush site in $\mathrm{PTEN}^{\mathrm{f} / \mathrm{f}}$ mice injected with either AAV-GFP or AAVCre (fig. S4A). However, at $3 \mathrm{dpc}$, axonal sprouts from PTEN-deficient RGCs began to penetrate the CSPG-enriched lesion site (fig. S4, B and D, and fig. S5A), and some fibers could be seen beyond the lesion sites $7 \mathrm{dpc}$ (figs. S4, C and E, and fig. S5B). In contrast, minimum axonal sprouts were seen in control animals at these stages (fig. S4, A to E, and fig. S5, A and B). Electron microscopic analysis confirmed that in wild-type mice, degenerating RGC axons, myelin debris, and macrophages occupied injury sites, and few regenerating fibers were visible (fig. S5, C and E). However, when PTEN was deleted in RGCs, regenerative axonal sprouts, often appearing as bundles, were found both within and distal to the lesion site soon after injury (fig. S5, D and F). These results indicate that PTEN deletion indeed enabled axons to overcome inhibition at the lesion site and to regenerate soon after injury.

PTEN deletion activates the PI3K/mTOR (mammalian target of rapamycin) pathway, which controls cell growth and size by regulating capdependent protein translation initiation (16, 17) (fig. S6). Two of the well-studied targets of the mTOR kinase are ribosomal S6 kinase 1 (S6K1) (which in turn phosphorylates ribosomal protein S6) and the eukaryotic initiation factor 4E (eIF4E)-binding protein 4E-BP1. We used antibodies to phospho-S6 (p-S6) to monitor the activity of the mTOR pathway in the RGCs. p-S6 staining revealed a development-dependent decline in the percentage of p-S6-positive RGCs (Fig. 2, A and B). Strong p-S6 signals can be seen in most embryonic neurons but are diminished in $90 \%$ of adult RGCs (Fig. 2, A and 
B), suggesting that mTOR signaling is down-regulated in the majority of adult RGCs, with only a small subset retaining considerable mTOR activity.

We next investigated whether axon injury alters the activity of the mTOR pathway and subsequent protein synthesis in adult RGCs. Upon experiencing stresses such as hypoxia, cells suppress the mTOR signaling (18-20), an evolutionally conserved stress response proposed to maintain energy homeostasis for survival. Thus, we postulated that the axotomy-triggered stress response may reduce global protein translation in injured neurons. To test this, we estimated the rate of new protein synthesis in purified RGCs from control and injured rats (figs. S7 and S8). Control and injured RGCs were incubated with ${ }^{35} \mathrm{~S}$-methanine/cysteine, and the results showed a significant decrease in new protein synthesis in axotomized RGCs (fig. S8).

We then studied whether axon injury suppressed the mTOR activity by immunohistochemistry of retinal sections with antibodies to p-S6. Axotomy almost completely abolished the remaining p-S6 signals in adult RGCs at all post-injury time points examined (1,3, and 7 days) in control mice (Fig. 3, A and B), indicating that axotomy triggers a rapid and sustained downregulation of the mTOR activity in all neurons. In cells under hypoxic stress, up-regulation of Redd1/2 (regulated in development and DNA damage responses $1 / 2$ ) is involved in the inhibition of mTOR (18-20). However, Redd1/2 mRNA levels were not altered in axotomized RGCs (fig. S9), suggesting that mechanisms other than Redd1/2 mediate the suppression of the mTOR pathway after axotomy.

We next assessed whether PTEN deletion affects the mTOR activity in the adult RGCs both before and after injury. AAV-Cre injection did not significantly increase the percentage of RGCs stained with antibodies to $\mathrm{p}-\mathrm{S} 6$ in uninjured PTEN ${ }^{\mathrm{f} / \mathrm{f}}$ mice, which may be due to the short interval between AAV-Cre injection and histological analysis (2 weeks). However, after optic nerve crush, similar percentages of p-S6-positive RGCs remained at 1, 3, or 7 days after injury in the AAV-Cre-injected PTEN ${ }^{\mathrm{f} / \mathrm{f}}$ mice (Fig. 3, A and B). Thus, despite experiencing a stress response to axotomy, these PTEN-deleted neurons were able to maintain mTOR activity levels similar to those of uninjured wild-type neurons. Notably, the percentage of regenerating fibers (8 to 10\%) was similar to that of p-S6-positive axotomized RGCs (Fig. 1E).

To further assess the relationship between p-S6-positive RGCs and RGCs that regenerated axons, we injected biotinylated dextran-amine (BDA), an axonal tracer, to the optic nerve region $\sim 2$ to $2.5 \mathrm{~mm}$ distal to the lesion site. This method resulted in few BDA-labeled cells in the retinas in wild-type mice at 14 days after injury (fig. S10A), consistent with the lack of regeneration of in wild-type animals. In PTEN-deleted animals, this same procedure labeled, on average, a total of 68 RGCs per retina, and $88 \%$ of these labeled RGCs were positively stained with antibodies to p-S6 (fig. S10). This result showed that the majority of regenerating fibers are from those p-S6-positive RGCs.

We next assessed the necessity of mTOR activation for the regeneration effects of PTENdeletion. Administration of rapamycin, an mTOR inhibitor, markedly reduced p-S6 signals in the RGCs (fig. S11, E and F) and largely neutralized the survival and regeneration observed in PTEN ${ }^{\mathrm{f} / \mathrm{f}}$ mice injected with AAV-Cre (fig. S11, A to D). Although residual axon regeneration observed in the PTEN-deleted mice treated with rapamycin may reflect possible involvements of other pathways downstream of PTEN, these results demonstrated a major role played by the mTOR activation in the effects of PTEN deletion.

To further examine whether activation of the mTOR pathway is sufficient to promote axon regeneration, we performed the optic nerve injury model in $\mathrm{TSCl}^{\mathrm{f} / \mathrm{f}}$ (tuberous sclerosis complex 1) conditional knockout mice (21). TSC1/2 form a protein complex that negatively regulates mTOR signaling, and loss of either TSC1 or TSC2 leads to constitutive activation of the mTOR pathway (22-24) (fig. S6). As expected, in AAV-Cre-injected $\mathrm{TSC1}^{\mathrm{f} / \mathrm{f}}$ mice, strong 
p-S6 signals were observed in axotomized RGCs (Fig. 4E), and RGC survival was enhanced after injury (Fig. 4, C and D). More important, considerable axon regeneration (Fig. 4, A and B) was observed in TSC1-deleted but not in wild-type mice injected with AAV-Cre. The extent of axon regeneration in TSC1-deleted mice was slightly weaker than that induced by PTEN deletion (comparing Fig. 1, A and E, to Fig. 4, A and B). Together with the observations that some p-S6-negative RGCs were labeled with BDA applied to the optic nerve distal to the lesion (fig. S10) and that the residual regeneration was observed in PTEN-deleted mice treated with rapamycin (fig. S11), these results suggest that changes in other downstream targets of PTEN, such as Akt and GSK-3 $(25,26)$, may also contribute to regenerative growth. Nonetheless, these data together indicate that activation of the mTOR pathway is sufficient to promote both RGC survival and axon regeneration.

Our findings reveal an important role for the regulation of the mTOR pathway and protein translation in determining the intrinsic axon-regrowth responsiveness of injured CNS neurons. This pathway is profoundly suppressed in axotomized adult neurons, which may limit new protein synthesis required for sustained axon regeneration. Reactivating the mTOR pathway by silencing either PTEN or TSC1 in adult neurons induces extensive axon regeneration, suggesting that retaining active protein synthesis in axotomized mature neurons is sufficient to initiate a neuronal regenerative program for axon regrowth. In light of the availability of specific chemical inhibitors of PTEN (27), these and other strategies may potentially be used transiently after CNS injury to prevent the down-regulation of protein synthesis and promote axon regeneration and functional recovery.

\section{References and Notes}

1. Schwab ME, Bartholdi D. Physiol. Rev 1996;79:319. [PubMed: 8618960]

2. Goldberg JL, Klassen MP, Hua Y, Barres BA. Science 2002;296:1860. [PubMed: 12052959]

3. Filbin MT. Philos. Trans. R. Soc. London B Biol. Sci 2006;361:1565. [PubMed: 16939975]

4. Fitch MT, Silver J. Exp. Neurol 2008;209:294. [PubMed: 17617407]

5. Case LC, Tessier-Lavigne M. Curr. Biol 2005;15:R749. [PubMed: 16169471]

6. Yiu G, He Z. Nat. Rev. Neurosci 2006;7:617. [PubMed: 16858390]

7. Harel NY, Strittmatter SM. Nat. Rev. Neurosci 2006;7:603. [PubMed: 16858389]

8. Weinberg, RA. The Biology of Cancer. Weinberg, RA., editor. Garland Science; 2007. p. 209-253.

9. Marino S, Vooijs M, van Der Gulden H, Jonkers J, Berns A. Genes Dev 2000;14:994. [PubMed: 10783170]

10. Yang X, Li C, Herrera PL, Deng C. Genesis 2002;32:80. [PubMed: 11857783]

11. Harfe BD, McManus MT, Mansfield JH, Hornstein E, Tabin CJ. Proc. Natl. Acad. Sci. U.S.A 2005;102:10898. [PubMed: 16040801]

12. Bardeesy N, et al. Nature 2002;419:162. [PubMed: 12226664]

13. Groszer M, et al. Science 2001;294:2186. [PubMed: 11691952]

14. Leon S, Yin Y, Nguyen J, Irwin N, Benowitz LI. J. Neurosci 2000;20:4615. [PubMed: 10844031]

15. Goldberg JL, et al. Neuron 2002;33:689. [PubMed: 11879647]

16. Guertin DA, Sabatini DM. Cancer Cell 2007;12:9. [PubMed: 17613433]

17. Luo J, Manning BD, Cantley LC. Cancer Cell 2003;4:257. [PubMed: 14585353]

18. Brugarolas J, et al. Genes Dev 2004;18:2893. [PubMed: 15545625]

19. Corradetti MN, Inoki K, Guan KL. J. Biol. Chem 2005;280:9769. [PubMed: 15632201]

20. Reiling JH, Hafen E. Genes Dev 2004;18:2879. [PubMed: 15545626]

21. Meikle L, et al. J. Neurosci 2007;27:5546. [PubMed: 17522300]

22. Garami A, et al. Mol. Cell 2003;11:1457. [PubMed: 12820960]

23. Inoki K, Zhu T, Guan KL. Cell 2003;115:577. [PubMed: 14651849]

24. Tee AR, et al. Curr. Biol 2003;13:1259. [PubMed: 12906785] 
25. Kim WY, et al. Neuron 2006;52:981. [PubMed: 17178402]

26. Namikawa K, et al. J. Neurosci 2000;20:2875. [PubMed: 10751440]

27. Rosivatz E, et al. ACS Chem. Biol 2006;1:780. [PubMed: 17240976]

28. We thank H. Hasegawa and F. Wang for providing Rosa-stop-PLAP mice, D. Kwiatkowski for proving $\mathrm{TSC}^{\mathrm{f} / \mathrm{f}}$ mice, C. Deng for Smad4 ${ }^{\mathrm{f} / \mathrm{f}}$ mice, M. Ericsson and L. Bu for microscopic analysis, and L. Benowitz, M. Greenberg, T. Hensch and J. Kanter for reading the manuscript. This study was supported by grants from Craig H. Neilson Foundation (to K.K.P.); National Institute of Neurological Disorders and Stroke (NINDS), Wings for Life, and Adelson Foundation (to Z.H.); Ruth L.

Kirschstein National Research Service Award (NRSA) (to Y.H.); Canadian Institute of Health Research (to P.D.S.); and NINDS and Tuberous Sclerosis Alliance (to M.S.). 


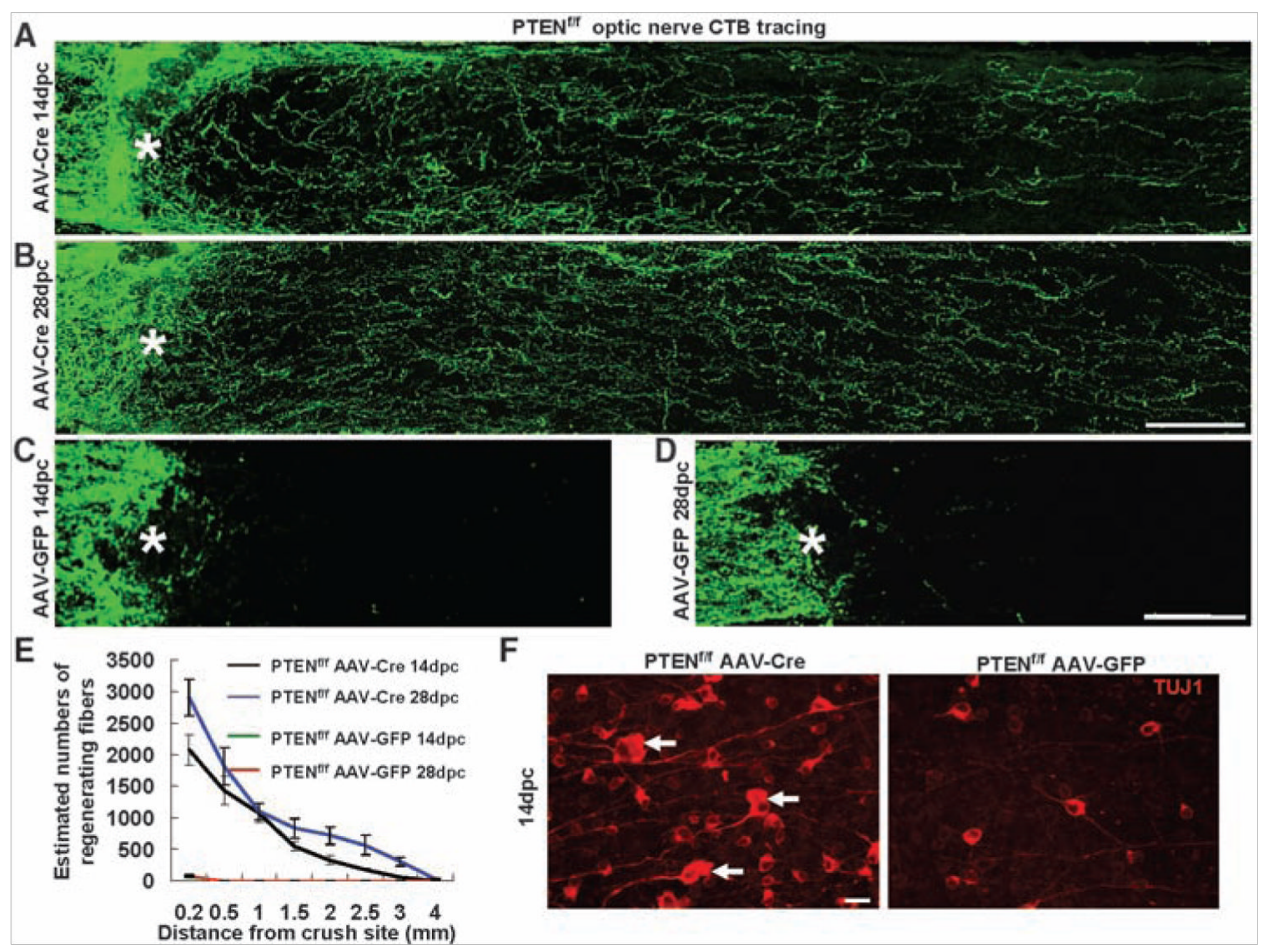

Fig. 1. PTEN deletion promotes RGC axon regeneration

(A to D) Confocal images of optic nerves showing CTB-labeled fibers around the lesion sites at 14 days [(A) and (C)] or 28 days [(B) and (D)] after injury from PTEN ${ }^{\mathrm{f} / \mathrm{f}}$ mice injected with AAV-Cre [(A) and (B)] or AAV-GFP [(C) and (D)]. Scale bar, $100 \mu \mathrm{m}$. (E) Quantification of regenerating fibers at different distances distal to the lesion sites. At least five different sections (every fourth section) from each animal were quantified. At both time points, there were significant differences between control and PTEN-deleted mice groups at every distance measured by analysis of variance with Bonferroni's post-test $[P<0.05$ for both $14 \mathrm{dpc}(n=7)$ and $28 \mathrm{dpc}(n=3)]$. (F) Fluorescent photomicrographs of retinal whole-mounts showing surviving TUJ1+ RGCs at 14 days after injury. Arrows, RGCs with enlarged soma; *, crush site; scale bar, $20 \mu \mathrm{m}$. 

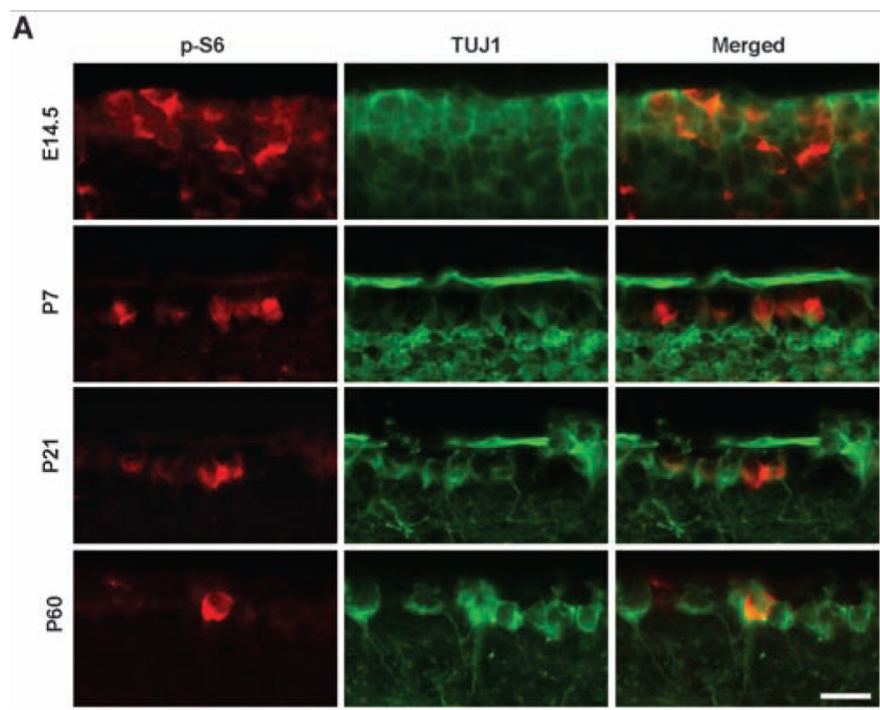

B

Fig. 2. Development-dependent decline of p-S6 signals in RGCs

Representative images (A) and quantification (B) of immunohistochemical analysis for p-S6 or TUJ1 immunoreactivity of the ganglion cell layer (GCL) of mouse retinas at different ages. Scale bar, $20 \mu \mathrm{m}$. Data are presented as mean percentages of $\mathrm{p}-\mathrm{S} 6^{+} \mathrm{TUJ} 1^{+}$cells among total $\mathrm{TUJ} 1^{+}$cells in the GCL of each retina. There is a significant difference in percentages of P21 and P60 when compared to that of P7. *, $P<0.01$, Student's $t$ test. Cell counts were performed on at least four nonconsecutive sections, from three mice per age group. 


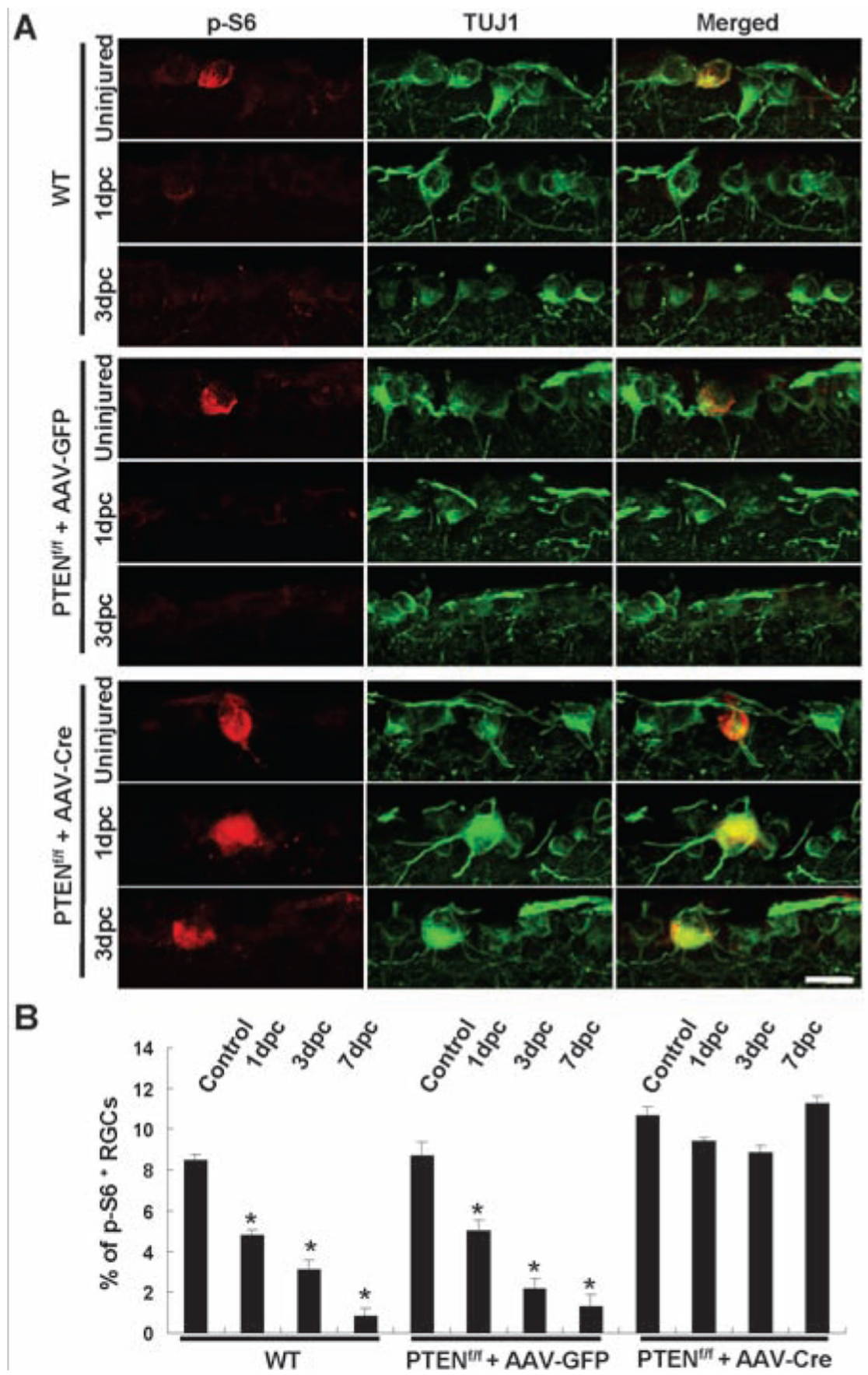

Fig. 3. Axotomy reduces p-S6 levels in RGCs in control but not in PTEN-deleted RGCs (A) Immunofluorescence analysis with antibodies to p-S6 or TUJ1 of the retinal sections from wild-type or PTEN ${ }^{\mathrm{f} / \mathrm{f}}$ mice injected with AAV-GFP or AAV-Cre in different animal groups. Scale bar, $20 \mu \mathrm{m}$. (B) Quantification of p-S6 ${ }^{+}$RGCs. Data are presented as mean percentages of $\mathrm{p}-\mathrm{S} 6^{+} \mathrm{TUJ} 1^{+}$cells among total $\mathrm{TUJ} 1^{+}$cells in the GCL of each retina. Cell counts were performed on at least four nonconsecutive sections, from three mice per group. ${ }^{*}, P<0.01$ by Dunnett's test. Comparisons were made against the uninjured control retinas. 


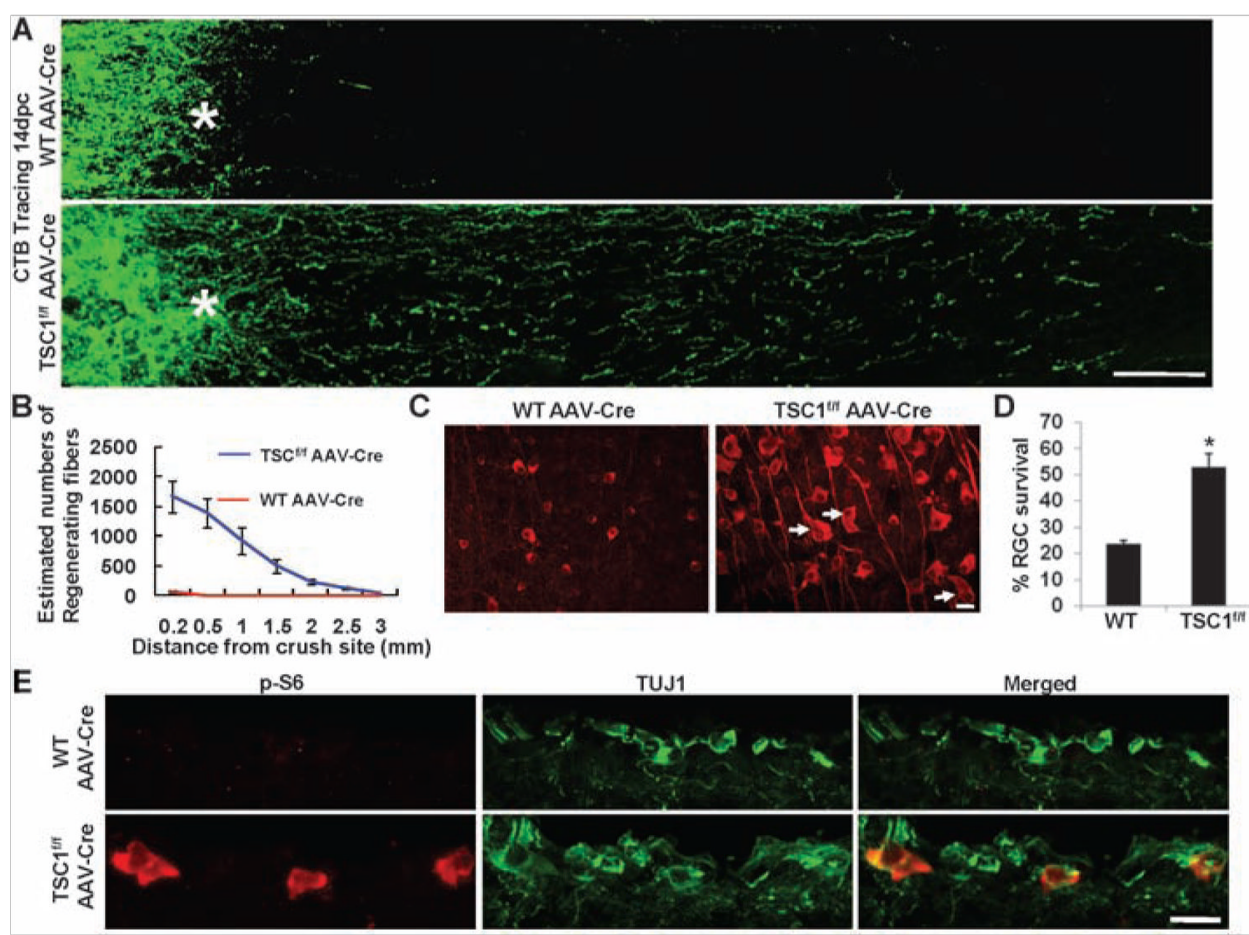

Fig. 4. TSC1 deletion promotes RGC survival and axon regeneration after optic nerve crush (A) Confocal images of optic nerves spanning lesion sites from wild-type or TSC1 ${ }^{\mathrm{f} / \mathrm{f}}$ mice injected with AAV-Cre at 14 days after crush. Scale bar, $100 \mu \mathrm{m}$. (B) Quantification of regenerating fibers in different groups. There is a significant difference between control and TSC1-deleted groups by Student's $t$ test at each distance $(P<0.05)$. (C) Fluorescent photomicrographs of retinal whole-mounts showing characteristic surviving $\mathrm{TUJ}{ }^{+} \mathrm{RGCs}$. Arrows, RGCs with enlarged soma; *, crush site; scale bar, $20 \mu \mathrm{m}$. (D) RGC survival at 14 dpc, expressed as a percentage of the total number of $\mathrm{TUJ} 1^{+} \mathrm{RGCs}$ in the uninjured retina. *, $P<0.05$, Student's $t$ test. (E) Immunofluorescence analysis of the retinal sections from AAVCre-injected wild-type or TSC $1^{\mathrm{f} / \mathrm{f}}$ mice at 14 days after injury with antibodies to $\mathrm{p}-\mathrm{S} 6$ or TUJ1. Scale bar, $20 \mu \mathrm{m}$. 